\title{
QUANTUM PROBABILITY SPACES
}

\author{
STANLEY P. GUDDER
}

1. Introduction. In [5] P. Suppes introduced the notion of a quantum probability space. He noted that such spaces may be used to describe the position and momentum of a quantum mechanical particle but cannot be used for more general systems. This author has considered quantum probability spaces not only because they are an interesting example of a nonclassical logic but because quantum mechanical phenomena are seen to develop in a quite transparent fashion in this case.

2. $\sigma$-classes and compatibility. Let $\Omega$ be a nonempty set. A $\sigma$-class $C$ of subsets of $\Omega$ is a collection of subsets which satisfy

(i) $\Omega \in C$;

(ii) if $a \in C$, then $a^{\prime} \in C$;

(iii) if $a_{i}$ are mutually disjoint, then $\bigcup a_{i} \in C, i=1,2, \cdots$;

where we have denoted the complement of a set $a$ by $a^{\prime}$. A state $m$ on $C$ is a map $m: C \rightarrow[0,1]$ such that

(i) $m(\Omega)=1$

(ii) if $a_{i}$ are mutually disjoint elements of $C$ then $m\left(\cup a_{i}\right)=\Sigma m\left(a_{i}\right)$. A quantum probability space is a triple $(\Omega, C, M)$ where $C$ is a $\sigma$-class of subsets of $\Omega$ and $M$ is the set of states on $C$.

Lemma 2.1. Let $C$ be a $\sigma$-class of subsets of $\Omega$.

(i) If $a, b \in C$ and $a \subset b$, then $b \cap a^{\prime} \in C$.

(ii) If $a_{1}, a_{2}, \cdots \in C$ and $a_{1} \subset a_{2} \subset a_{3} \subset \cdots$, then $\bigcup a_{i} \in C$.

(iii) If $a_{1}, a_{2}, \cdots \in C$ and $a_{1} \supset a_{2} \supset a_{3} \supset \cdots$, then $\cap a_{i} \in C$.

Proof. (i) Since $a \cap b^{\prime}=\varnothing$ we have that $a \cup b^{\prime} \in C$. Hence $b \cap a^{\prime}$ $=\left(a \cup b^{\prime}\right)^{\prime} \in C$. (ii) By (i) $a_{2} \cap a_{1}^{\prime}, a_{3} \cap a_{2}^{\prime}, \ldots \in C$. Since these last sets are mutually disjoint we have $\cup a_{i}=a_{1} \cup\left(a_{2} \cap a_{1}^{\prime}\right) \cup\left(a_{3} \cap a_{2}^{\prime}\right)$ $\cup \ldots \in C$. (iii) This follows from (ii) by complementation.

If $a \subset b$ we use the notation $b-a=b \cap a^{\prime}$. We next show that there is an abundance of states on any $\sigma$-class. A state $m$ is concentrated at a point $\omega \in \Omega$ if $m(a)=1$ if $\omega \in a$ and $m(a)=0$ if $\omega \notin a$.

Lemma 2.2. If $(\Omega, C, M)$ is a quantum probability space and $a, b \in C$, then $a \subset b$ if and only if $m(a) \leqq m(b)$ for all $m \in M$.

PRoof. If $a \subset b$, then $b=a \cup(b-a)$ where $a$ and $b-a$ are disjoint.

Received by the editors June 26, 1968. 
Thus $m(b)=m(a)+m(b-a) \geqq m(a)$ for all $m \in M$. If $m(a) \leqq m(b)$ for all $m \in M$, by considering states concentrated on $a$, we see that $a \subset b$.

The motivation for this theory is, of course, quantum mechanics. Physically $C$ corresponds to a class of quantum mechanical events. In quantum theory the experiments used to verify two events $a, b \in C$ may interfere and it may be meaningless to consider $a$ and $b$ at the same time. However, if $a \cap b=\varnothing$ then there is no danger of interference so we may consider their logical join $a \cup b$, which is why we defined a $\sigma$-class as we did. If $a$ and $b$ are not disjoint, then they may be incompatible in the sense that no experiment can verify $a \cap b$ or $a \cup b$. Motivated by this we say that $a, b \in C$ are compatible (written $a \leftrightarrow b)$ if $a \cap b \in C$. Notice that if $a \subset b$ or if $a \cap b=\varnothing$ then $a \leftrightarrow b$.

LemмA 2.3. Let $C$ be $a$-class. (i) If $a, b \in C$ and $a \leftrightarrow b$, then $a \leftrightarrow b^{\prime}$ and $a \cup b \in C$. (ii) Suppose $a, a_{1}, a_{2}, \cdots \in C, a \leftrightarrow a_{i}, i=1,2, \cdots, \cup a_{i}$ and $\mathrm{U}\left(a \cap a_{i}\right) \in C$. Then $a \leftrightarrow \bigcup a_{i}$.

Proof. (i) Since $a \cap b \subset a$ by Lemma 2.1(i), $a-(a \cap b) \in C$. But $a \cap b^{\prime}=a-(a \cap b)$ so $a \leftrightarrow b^{\prime}$. Also

$$
a \cup b=[a-(a \cap b)] \cup(a \cap b) \cup[b-(a \cap b)]
$$

and since the latter sets are mutually disjoint $a \cup b \in C$.

(ii) By the distributive law $a \cap\left(\bigcup a_{i}\right)=\mathrm{U}\left(a \cap a_{i}\right) \in C$.

A $\sigma$-field of subsets $F$ of a nonempty set $\Omega$ is a class of subsets that satisfies

(i) $\Omega \in F$;

(ii) if $a \in F$, then $a^{\prime} \in F$;

(iii) if $a_{i} \in F, i=1,2, \cdots$, then $\bigcup a_{i} \in F$.

If (iii) holds for finite unions, $F$ is called a field.

Lemma 2.4. $A$ o-class $C$ is a $\sigma$-field if and only if all its elements are compatible.

Proof. Necessity is trivial. If all the elements of $C$ are compatible then finite unions of elements in $C$ are in $C$ by Lemma 2.3(i). If $a_{i}$ is a sequence in $C$, then

$$
\cup a_{i}=a_{1} \cup\left(a_{2}-a_{1} \cap a_{2}\right) \cup\left(a_{3}-\left(a_{1} \cup a_{2}\right) \cap a_{3}\right) \cup \cdots \in C .
$$

If $A_{1}, A_{2} \subset C$ we write $A_{1} \leftrightarrow A_{2}$ if $a_{1} \leftrightarrow a_{2}$ for all $a_{1} \in A_{1}, a_{2} \in A_{2}$. The sub $\sigma$-fields of a $\sigma$-class are extremely important because they correspond to classical experiments and single quantum mechanical quantities. It is important to know when a collection of sub $\sigma$-fields is contained in a single sub $\sigma$-field. 
Theorem 2.5. If $F_{1}$ and $F_{2}$ are sub $\sigma$-fields of $a$-class $C$ there is a sub $\sigma$-field containing them if and only if $F_{1} \leftrightarrow F_{2}$.

Proof. Necessity is trivial. For sufficiency let us first assume that $F_{1} \leftrightarrow F_{2}$ and that $F_{1}$ and $F_{2}$ have a finite number of elements. Let $\left\{a_{i}\right\}_{1}^{p}$ and $\left\{b_{i}\right\}_{1}^{q}$ be the distinct nonempty minimal elements of $F_{1}$ and $F_{2}$ respectively. Then clearly the $a_{i}$ 's are mutually disjoint (so are the $b_{i}^{\prime}$ 's), and $U a_{i}=\Omega\left(U b_{i}=\Omega\right)$. Let $c_{i j}=a_{i} \cap b_{j}, i=1,2, \cdots, p$, $j=1,2, \cdots, p$. Clearly the $c_{i j}$ 's are mutually disjoint and $U_{j} c_{i j}$ $=\bigcup\left(a_{i} \cap b_{j}\right)=a_{i} \cup\left(\cap b_{j}\right)=a_{i}, U_{i} c_{i j}=b_{j}$, and $U_{i, j} c_{i j}=\Omega$. If $F_{1} \vee F_{2}$ is $\varnothing$ together with all unions of the $c_{i j}$ 's then $F_{1}, F_{2} \subset F_{1} \bigvee F_{2}$ and $F_{1} \vee F_{2}$ is a sub $\sigma$-field with a finite number of elements. Now assume that $F_{1}$ and $F_{2}$ are arbitrary sub $\sigma$-fields and $F_{1} \leftrightarrow F_{2}$. Let $F=\bigcup A_{1} \bigvee A_{2}$ where $A_{1}, A_{2}$ run over all finite sub $\sigma$-fields such that $A_{1} \subset F_{1}, A_{2} \subset F_{2}$. Clearly, $F_{1} \subset F$ and $F_{2} \subset F$. Now $\varnothing, \Omega \in F$ and if $a \in F$ then $a^{\prime} \in F$. If $a, b \in F$ then there is an $A_{1}, A_{2}, A_{1}^{0} A_{2}^{0}$ such that $a \in A_{1} \vee A_{2}, b \in A_{1}^{0} \vee A_{2}^{0}$. Let ting $A_{1}^{00}=A_{1} \bigvee A_{1}^{0}$ and $A_{2}^{00}=A_{2} \bigvee A_{2}^{0}$, we see that $a$ and $b$ are in $A_{1}^{00} \vee A_{2}^{00}$. Thus $a \leftrightarrow b$, and $a \cup b \in F$. Thus $F$ is a subfield of $C$. The smallest monotone class of sets including $F$ is a $\sigma$-field $[2$, p. 27] and is included in $C$ by Lemma 2.1 .

3. c-classes and internal compatibility. Let us now consider some examples.

EXAmple 1. Let $\Omega=\{1,2,3,4,5,6\}$ and let $C$ be the class of subsets of $\Omega$ with an even number of elements. Then $C$ is a $\sigma$-class of subsets but is not a $\sigma$-field since $\{1,2\} \cap\{2,3\}=\{2\} \notin C$, for instance. We thus see, of course, that $\{1,2\} \leftrightarrow\{2,3\}$.

We have seen in Theorem 2.5 that if $F_{1}$ and $F_{2}$ are compatible sub $\sigma$-fields of a $\sigma$-class then they are contained in a common sub $\sigma$-field. This result does not hold for three sub $\sigma$-fields, as the next example shows.

Example 2. Let $\Omega=\{1,2, \cdots, 8\}$ and let $C$ be the class of subsets of $\Omega$ with an even number of elements. Then again $C$ is a $\sigma$-class but not a $\sigma$-field. Let $a=\{1,2,3,4\}, b=\{1,2,5,6\}$, and $c=\{1,3,6,8\}$. Then $a, b$ and $c$ are mutually compatible and are thus contained in three mutually compatible sub $\sigma$-fields $F_{1}, F_{2}$ and $F_{3}$ respectively. However, we see that $a \cup b \leftrightarrow \rightarrow c$ and hence there is no sub $\sigma$-field containing $F_{1}, F_{2}$ and $F_{3}$.

We can get around the difficulty in Example 2 by making the following definition. If $\Omega$ is a nonempty set, a $c$-class ( $c$ is for compatibility) $C$ of subsets of $\Omega$ is a $\sigma$-class which satisfies the following: If $a, b, c$ are mutually compatible sets in $C$, then $c \leftrightarrow a \cup b$. From the physical point of view it is reasonable to assume that quantum events 
satisfy this postulate. One can show that the $\sigma$-class in Example 1 is a $c$-class but clearly the $\sigma$-class in Example 2 is not. Of course a $\sigma$-field is a $c$-class. As we shall see $c$-classes are much better behaved than $\sigma$-classes.

If $C$ is a $\sigma$-class and $A \subset C$, then the compatant of $A$ is $A^{\circ}$ $=\{b \in C: b \leftrightarrow a$ for all $a \in A\}$. Clearly $A_{1} \subset A_{2}$ implies $A_{2}^{c} \subset A_{1}^{c}$. Denoting $\left(A^{c}\right)^{c}$ by $A^{c c}$ it is clear that $A \subset A^{c c} . A$ set $A \subset C$ is compatible if the elements of $A$ are mutually compatible and obviously $A \subset A^{c}$ if and only if $A$ is compatible.

Theorem 3.1. Let $C$ be a $\sigma$-class in $\Omega$. Every compatible subset of $C$ is contained in a sub $\sigma$-field if and only if $C$ is a c-class.

Proof. Necessity is trivial. To prove sufficiency suppose $C$ is a $c$-class and $A \subset C$ is compatible. Since $A \subset A^{c c}$, it suffices to show that $A^{c c}$ is a sub $\sigma$-field. Obviously $\varnothing, \Omega \in A^{c c}$ and if $a \in A^{c c}$ then $a^{\prime} \in A^{c c}$. Now since $A \subset A^{c}$ we have that $A^{c c} \subset\left(A^{c c}\right)^{c}$ and thus $A^{c c}$ is compatible. Let $a_{i}$ be a sequence of elements of $A^{c c}$. As in the proof of Lemma 2.4, $\cup a_{i} \in C$ (here we use the fact that $C$ is a $c$-class). If $b \in A^{c}$, then $b \leftrightarrow a_{i}$, $i=1,2, \cdots$, and since $b \cap a_{i} \leftrightarrow b \cap a_{j}$, we have $U\left(b \cap a_{i}\right) \in C$ as in the previous sentence. Hence by Lemma 2.3(ii), $b \leftrightarrow \bigcup a_{i}$ and $\bigcup a_{i} \in A^{\text {cc }}$ which completes the proof.

Corollary 3.2 If $C$ is a c-class and $\left\{F_{\lambda}\right\}$ a collection of mutually compatible sub $\sigma$-fields, then $U F_{\lambda}$ is contained in a sub $\sigma$-field.

One might expect, in view of Theorem 3.1 and Lemma 2.4, that if we had some compatible elements in a $c$-class then the smallest sub $c$-class containing these elements would be a sub $\sigma$-field. (Or the corresponding statement about two sub $\sigma$-fields in a $\sigma$-class.) However, these statements are not true. For instance, consider Example 1. Let $C_{1}$ be the class of all subsets of $\Omega$ and let $C$ be the $\sigma$-class of that example. Then the elements of $C$ are certainly compatible relative to $C_{1}$, however the smallest sub $c$-class containing $C$ is $C$ itself which is certainly not a sub $\sigma$-field of $C_{1}$.

The previous paragraph emphasizes the fact that compatibility is defined relative to a certain $\sigma$-class and that elements compatible relative to one $\sigma$-class obviously need not be compatible relative to another. This is not the case, however, in the following situation. A class of subsets $A$ of a nonempty set $\Omega$ is internally compatible if $a \cap b \in A$ for all $a, b \in A$. Thus if $A$ is internally compatible it is compatible relative to any $\sigma$-class containing it.

Corollary 3.3. If $A$ is internally compatible then the c-class $C$ generated by $A$ (i.e. the smallest c-class containing $A$ ) is a $\sigma$-field. 
Proof. Since $A$ is compatible relative to $C$, applying Theorem 3.1 there is a sub $\sigma$-field $F$ containing $A$. But $F$ is a $c$-class and by the minimality of $C$ we have $C=F$.

Corollary 3.3 does not hold if we replace the word $c$-class by $\sigma$-class. Indeed, in Example 2, let $A=\{a, b, c,\{1,2\},\{1,3\},\{1,6\},\{1\}\}$. Then $A$ is internally compatible and $C$ in Example 2 is a $\sigma$-class containing $A$. Hence the $\sigma$-class $C_{1}$ generated by $A$ is contained in $C$. But since $c \cap(a \cup b) \notin C$ then $c \cap(a \cup b) \notin C_{1}$ and hence $C_{1}$ cannot be a $\sigma$-field.

We now give an application of Corollary 3.3. Let $\Omega$ be a topological space and let $A$ be the class of open subsets of $\Omega$. Since the intersection of two open sets is open, $A$ is internally compatible. Hence the $c$-class generated by $A$ is a $\sigma$-field which is the usual Borel field. Thus in topological spaces if one wants to consider all the open sets then nothing new is gained by using $c$-classes. The author does not know whether the $\sigma$-class $C$ generated by $A$ is the Borel field, although $C$, of course, is contained in it. However, $C$ is certainly quite large. For example, if $\Omega$ is the real line $R$ then it is easily seen that $C$ contains all the $G \delta$ 's and $F_{\sigma}$ 's and thus $C$ differs from the Borel field at most by sets of Lebesgue measure zero.

4. Observables. Let us now consider what corresponds in this theory to measurable functions. If $(\Omega, C, M)$ is a quantum probability space, a real valued function $f: \Omega \rightarrow R$ is observable if $f^{-1}(E) \in C$ for every set $E$ in the Borel field $B(R)$. Observable functions are called $o b$ servables. If $f$ is observable we use the notation $A_{f}=\left\{f^{-1}(E): E \in B(R)\right\}$. It is easily seen that $A_{f}$ is a sub $\sigma$-field. We say that two observables $f$ and $g$ are compatible (written $f \leftrightarrow g$ ) if $A_{f} \leftrightarrow A_{g}$. It is clear that if $g$ is a real Borel function and $f$ an observable then $g \circ f$ is observable. Also if $f_{1} \leftrightarrow f_{2}$ and $g_{1}, g_{2}$ are real Borel functions, then $g_{1} \circ f_{1} \leftrightarrow g_{2} \circ f_{2}$.

THEOREM 4.1. Let $C$ be a $\sigma$-class. (i) If $a, b \in C$, then $a \leftrightarrow b$ if and only if there is an observable $f$ such that $a, b \in A_{f}$. (ii) If $f$ and $g$ are observable and $f \leftrightarrow g$, then $f+g$ and $f \cdot g$ are observable. (iii) $C$ is a $\sigma$-field if and only if the sum of any two observables is observable.

Proof. (i) If $f$ is observable and $a, b \in A_{f}$, then $a \leftrightarrow b$ since $A_{f}$ is a sub $\sigma$-field. If $a \leftrightarrow b$ define the function $f$ by $f(\omega)=0$ for $\omega \in a \cap b$, $f(\omega)=1$ for $w \in a-a \cap b, f(\omega)=2$ for $\omega \in b-a \cap b$, and $f(\omega)=3$ for $\omega \in a^{\prime} \cap b^{\prime}$. Then $f$ is observable and $a=f^{-1}(\{0,1\}), b=f^{-1}(\{0,2\})$ and hence $a, b \in A_{f}$. (ii) Since $f \leftrightarrow g$, we have $A_{f} \leftrightarrow A_{g}$. By Theorem 2.5 $A_{f} \cup A_{g}$ is contained in a sub $\sigma$-field and the result follows from the usual measure theoretic argument. (iii) If $C$ is a $\sigma$-field, then all ob- 
servables are mutually compatible and the result follows from (ii). Now suppose the sum of any two observables is observable and $a, b \in C$. If $\chi_{a}$ and $\chi_{b}$ denote the characteristic functions of $a$ and $b$ respectively then, $\chi_{a}+\chi_{b}$ is observable and hence $a \cap b$ $=\left(\chi_{a}+\chi_{b}\right)^{-1}(\{2\}) \in C$. Thus $a \leftrightarrow b$ and by Lemma $2.4 C$ is a $\sigma$-field.

It follows from Theorem 4.1 that the sum of two noncompatible observables need not be observable. However, there are noncompatible observables whose sums are observable. For instance, in Example 1 define the functions $f$ and $g$ by $f(1)=f(2)=0, f(3)=f(4)=1$, $f(5)=f(6)=2 ; g(1)=g(6)=1, g(2)=g(4)=2, g(3)=g(5)=0$. Then $f \longleftrightarrow g$ since $f^{-1}(\{0\}) \cap g^{-1}(\{1\})=\{1\} \notin C$ for instance. Now it is easy to check that $f+g$ and $f \cdot g$ are observable. However, $f-g$ is not observable! Notice also that $f+g$ and $f \cdot g$ are not compatible with $f$ or $g$. This type of behavior cannot occur with characteristic functions.

LEMMA 4.2. If $C$ is a $\sigma$-class and $a, b \in C$, then the following statements are equivalent. (i) $a \leftrightarrow b$; (ii) $\chi_{a} \leftrightarrow \chi_{b}$; (iii) $\chi_{a}+\chi_{b}$ is observable; (iv) $\chi_{a} \cdot \chi_{b}$ is observable; (v) $\chi_{a}-\chi_{b}$ is observable.

We leave the simple proof to the reader.

Let us now briefly consider integration in this theory. If $f$ is observable and $m$ a state then $A_{f}$ is a $\sigma$-field and $m$ is a measure on $A_{f}$. Thus $\left(\Omega, A_{f}, m\right)$ becomes an ordinary probability space and we can define the integral $\int f d m$ in the usual way.

LemMA 4.3. If $f$ and $g$ are (not necessarily compatible) bounded observables and $\int f d m=\int g d m$ for every state $m$, then $f=g$.

ProOF. If $m$ is concentrated at a point $\omega_{0}$ and $s$ is a simple function, it follows that $\int s d m=s\left(\omega_{0}\right)$. Since $f$ is a limit of simple functions we see that $\int f d m=f\left(\omega_{0}\right)$.

We thus see that bounded observables satisfy the uniqueness condition discussed in [1]. Another natural question is whether integration is linear. That is, does $\int(f+g) d m=\int f d m+\int g d m$ for $f, g$ bounded observables whose sum is observable and $m$ any state? The author does not know the answer to this question; however, it is trivial that the answer is affirmative for states concentrated at points. The answer is also affirmative if $f$ and $g$ are simple functions.

5. Comparison with Mackey's theory. Let us now compare our theory to that given by Mackey [3] in his axiomatic development of quantum mechanics. In the sequel we will always assume $C$ is a $\sigma$-class. Now $C$ may be regarded as a complemented partially ordered 
set in which we define $a \leqq b$ if $a \subset b$ and use the usual set complementation. The sup and inf $a \bigvee b, a \wedge b$, respectively, are defined in the usual way relative to $C$. Note however that $a \bigvee b(a \wedge b)$ need not equal $a \cup b(a \cap b)$ even if the former exist. (They are equal if the latter are in C.) For instance, in Example $1\{1,2\} \wedge\{2,3\}=\varnothing$ while $\{1,2\} \cap\{2,3\}=\{2\} \neq \varnothing$. This also shows that if $a \wedge b=\varnothing$ then $a$ and $b$ need not be disjoint $(a \cap b=\varnothing)$. Thus $a \leftrightarrow b$ is not equivalent to $a \wedge b \in C$ although the former does imply the latter. We say that $a, b \in C$ split if there exist mutually disjoint elements $a_{1}, b_{1}, c \in C$ such that $a=a_{1} \vee c$ and $b=b_{1} \vee c$. Mackey calls this simultaneous answerability and it corresponds to our notion of compatibility.

Theorem 5.1. Suppose $a, b \in C$. (i) If $a \leftrightarrow b$ then $a \bigvee b=a \cup b$ and $a \wedge b=a \cap b$. (ii) $a \leftrightarrow b$ if and only if $a$ and $b$ split.

Proof. (i) If $a \leftrightarrow b$ then $a \cap b \in C$ and then clearly $a \cap b=a \wedge b$. (ii) Suppose $a$ and $b$ split and $a=a_{1} \bigvee c, b=b_{1} \bigvee c$ where $a_{1}, b_{1} c$ are mutually disjoint elements of $C$. By (i) $a=a_{1} \cup c$ and $b=b_{1} \cup c$. Thus $c \subset a \cap b$. If $a \cap b=\varnothing$ we are finished. Otherwise suppose $\omega \in a \cap b$. Then $\omega \in c$ or $\omega \in b_{1}$. Suppose $\omega \in b_{1}$. Then $\omega \notin c, \omega \notin a_{1}$ and hence $\omega \notin a$, a contradiction. Hence $a \cap b=c \in C$ and $a \leftrightarrow b$. Conversely, if $a \leftrightarrow b$ then $a \cap b \in C$ and $a-a \cap b, b-a \bigcap b \in C$. Thus $a=(a-a \cap b) \vee(a \cap b)$ and $b=(b-a \cap b) \vee(a \cap b)$.

Using this theorem we see that our language can be translated into the language in Mackey's theory and that our Theorems 2.5, 4.1(i) and 3.1 are related to the results of Varadarajan [6], [7], and Pool [4] respectively. It is left to the reader to find the exact relation between these theories.

\section{REFERENCES}

1. S. P. Gudder, Uniqueness and existence properties of bounded observables, Pacific J. Math. 19 (1966), 81-93, 588-589.

2. P. R. Halmos, Measure theory, Van Nostrand, Princeton, N. J., 1950.

3. G. W. Mackey, The mathematical foundations of quantum mechanics, Benjamin, New York, 1963.

4. J. C. T. Pool, Simultaneous observability and the logic of quantum mechanics, Ph.D. Thesis, State University of Iowa, Iowa City, Iowa, 1963.

5. P. Suppes, The probabilistic argument for a non-classical logic for quantum mech anics, Philos. Sci. 33 (1966), 14-21.

6. V. S. Varadarajan, Probability in physics and a theorem on simultaneous observability, Comm. Pure Appl. Math. 15 (1962), 189-217; correction, loc. cit. 18 (1965).

7. - Geometry of quantum theory, Vol. 1, Van Nostrand, Princeton, N. J., 1968.

UNIVERSITY OF WISCONSIN 